\title{
Chondrogenic differentiation of human adipose-derived stem cells using microcarrier and bioreactor combination technique
}

\author{
HONGJUN KANG ${ }^{1}$, SHIBI LU ${ }^{1}$, JIANG PENG ${ }^{1}$, QIANG YANG ${ }^{2}$, SHUYUN LIU ${ }^{1}$, LI ZHANG ${ }^{1}$, \\ JINGXIANG HUANG ${ }^{1}$, XIANG SUI ${ }^{1}$, BIN ZHAO ${ }^{1}$, AIYUAN WANG ${ }^{1}$, \\ WENJING XU ${ }^{1}$, QUANYI GUO ${ }^{1}$ and QING SONG $^{3}$ \\ ${ }^{1}$ Key Laboratory of PLA, Department of Orthopaedics, Chinese PLA General Hospital, Beijing 100853; \\ ${ }^{2}$ Department of Spine Surgery, Tianjin Hospital, Hexi, Tianjin 300211; ${ }^{3}$ Critical Care Medicine, \\ Chinese PLA General Hospital, Beijing 100853, P.R. China
}

Received December 7, 2013; Accepted September 18, 2014

DOI: $10.3892 / \mathrm{mmr} .2014 .2820$

\begin{abstract}
The aim of the current study was to explore the application of microcarrier technology in the rapid amplification and chondrogenic differentiation of human adipose-derived stem cells (ADSCs) in a rotating bioreactor. Human ADSCs were cultivated with Cytodex 3 microcarriers in a rotary cell culture system (RCCS), and using inverted and scanning electron microscopes, the ADSCs were observed on the surface of the microcarriers. The harvested ADSCs were stained with safranin-O or toluidine blue histochemical stains, and type II collagen immunohistochemical stain. ADSCs were adherent to the surface of Cytodex 3 microcarriers by $24 \mathrm{~h}$. They became short and spindle-shaped, and as time progressed, the adherence of the cells to the microcarriers gradually improved. By the end of the culture period, the cell densities were $\sim 19$ times that of the initial cell density. The harvested cells on microcarriers were safranin-O and toluidine blue staining and collagen II-positive staining, which were stronger than the control group. The application of microcarrier technology is able to rapidly amplify human ADSC proliferation and successfully implement chondrogenic differentiation in vitro.
\end{abstract}

\section{Introduction}

Since Zuk et al (1) extracted pluripotent stem cells from fat in 2001, adipose-derived stem cells (ADSCs) have become a focus of research into tissue engineering, due to their multiple

Correspondence to: Dr Quanyi Guo, Key Laboratory of PLA, Department of Orthopaedics, Chinese PLA General Hospital, 28 Fuxing Road, Beijing 100853, P.R. China

E-mail: quanyiguodoc@163.com

Dr Qing Song, Critical Care Medicine, Chinese PLA General Hospital, 28 Fuxing Road, Beijing 100853, P.R. China

E-mail: doctorklbd@126.com

Key words: adipose-derived stem cells, bioreactor, microcarrier, cartilage cells advantages, including extensive sources, convenient extraction methods and the ability to be rapidly amplified. A number of studies have confirmed that ADSCs can differentiate into multiple cell types in vitro, including bone, cartilage, fat, muscle, neural and endothelial cells (2-6), indicating characteristics similar to bone marrow stromal stem cells and a prospective application in tissue engineering. Furthermore, cartilage is recognized as the most suitable tissue for construction by tissue engineering, thus ADSCs have great potential as seed cells for cartilage tissue engineering. Cartilage tissue engineering requires large quantities of seed cells in the short-term, which can maintain a chondrocyte phenotype in the process of in vitro culture.

It is difficult to meet these requirements with traditional static culture, whilst three-dimensional dynamic culture is able to accelerate cell proliferation and be conducive to chondrogenic differentiation and phenotype maintenance (7). Therefore, the present study applied bioreactor technology combined with microcarriers to rapidly amplify and induce chondrogenic differentiation in ADSCs. The aim was to investigate the proliferation and differentiation of ADSCs on microcarriers, and explore the feasibility of clinical application in the future.

\section{Materials and methods}

Materials. Type I collagenase, dexamethasone, 2-phospho-Lascorbic acid (vitamin C), ITS liquid media supplement and Cytodex 3 microcarrier beads (Sigma-Aldrich, Poole, UK); trypsin, Dulbecco's modified Eagle's medium (DMEM)-high glucose (Gibco Life Technologies, Carlsbad, CA, USA); fetal bovine serum (FBS; Beijing Yuan Heng Sheng Ma Biotechnology Institute, Beijing, China); recombinant murine FGF-basic and TGF- $\beta 1$ (PeproTech EC Ltd., London, UK); type II collagen mouse anti-human monoclonal antibody (Beijing Zhongshan Co., Beijing, China); a rotating bioreactor (Synthecon, Inc., Houston, TX, USA); and an inverted microscope (Olympus IX70; Olympus Corporation, Tokyo, Japan).

Isolation and culture of ADSCs. The present study and the collection of human fat tissue was under the ethical approval 
of the Chinese PLA General Hospital Ethics Committee (Beijing, China). The infrapatellar fat pad removed during knee arthroplasty was maintained in strictly aseptic conditions, and stem cells were separated within $30 \mathrm{~min}$ of harvest. The superficial fascia and small blood vessels on the surface of the fat tissue were carefully removed with microsurgical scissors, D-Hank's solution was used to wash the samples three times to remove red cells. The fat was cut into pieces, added to $5 \mathrm{X} 0.075 \%$ type I collagenase, stirred for $30 \mathrm{~min}$ at $37^{\circ} \mathrm{C}$ and centrifuged at $671 \mathrm{x} \mathrm{g}$ for $5 \mathrm{~min}$ to release the fat and the supernatant. It was then washed again with D-Hank's solution, filtered through a 100-mesh filter (BD Biosciences, Suzhou, China) and centrifuged at $168 \mathrm{x}$ g for $5 \mathrm{~min}$. Then the precipitation was washed twice in D-Hank's solution, which was prepared in the Key Laboratory of PLA (Chinese PLA General Hospital, Beijing, China) and contained $\mathrm{KCl}(0.4 \mathrm{~g} / \mathrm{l}), \mathrm{KH}_{2} \mathrm{PO}(0.06 \mathrm{~g} / \mathrm{l}), \mathrm{NaCl}$ $(8.0 \mathrm{~g} / \mathrm{l}), \mathrm{NaHCO}_{3}(0.35 \mathrm{~g} / \mathrm{l}), \mathrm{Na}_{2} \mathrm{HPO}_{4} .7 \mathrm{H}_{2} \mathrm{O}(0.06 \mathrm{~g} / \mathrm{l})$ and phenol red $(0.02 \mathrm{~g} / \mathrm{l})$. The cells were then resuspended in $10 \%$ FBS containing high-glucose DMEM medium, with a change of medium after $48 \mathrm{~h}$. When the cells were at $80 \%$ confluency, $0.25 \%$ trypsin was used for digestion passage culture. At the second passage, the medium was changed to cartilage-inducing liquid $(5 \mathrm{ng} / \mathrm{ml}$ FGF-2; $10 \mathrm{ng} / \mathrm{ml}$ TGF- $\beta 1 ; 50 \mu \mathrm{g} / \mathrm{ml}$ vitamin C; $10^{-7} \mathrm{M}$ dexamethasone; and 1X ITS) for cartilage differentiation.

Pretreatment with Cytodex 3. The dry weight of $50 \mathrm{mg}$ microcarriers was placed into $10-\mathrm{ml}$ clean glass bottles, with $10 \mathrm{ml}$ phosphate-buffered saline (PBS; Sigma-Aldrich) without calcium and magnesium ions, and incubated at room temperature for at least $3 \mathrm{~h}$ to expand the microcarriers. The supernatant was discarded with a polyethylene aluminum compound pipe straw, the microcarriers were cleaned twice with PBS without calcium and magnesium ions, which was then discarded prior to the addition of $10 \mathrm{ml}$ fresh PBS without calcium and magnesium ions. The samples then underwent high pressure sterilization for $20 \mathrm{~min}$, the supernatant liquid was removed and the microcarriers were cleaned with culture liquid. They were then stored in a refrigerator at $4^{\circ} \mathrm{C}$.

Cell culture methods. The Cytodex 3 microcarriers $(50 \mathrm{mg})$ and ADSCs (final concentration, $1 \times 10^{5} / \mathrm{ml}$ ) were added to a $10-\mathrm{ml}$ rotary cell culture system (RCCS; Synthecon, Inc., Houston, TX, USA) culture container, then the cartilage inducing medium was added to fill the container. The container with cell-microcarriers complex was connected to the rotating base. The device was put in a $5 \% \mathrm{CO}_{2}$ incubator and was cultured at $37^{\circ} \mathrm{C}$. In order to fully mix cells, the container was rotated at a force of $5 \mathrm{rpm}$ for $5 \mathrm{~min}$ and paused for $5 \mathrm{~min}$ alternately, which was repeated for several cycles, it was then continuously rotated and gradually adjusted to 10-12 rpm. Once the culture medium exhibited a yellow color and a PH value $<7.0$, the medium was changed. Static culture was conducted by seeding $1 \times 10^{5} / \mathrm{ml}$ ADSCs into five wells of a 6-well plate, $2 \mathrm{ml}$ in each well. During the culture, the medium was changed every 2-3 days to maintain a sufficient quantity of cells.

Cell morphological observation and counting. Human ADSCs were cultured for 1, 3, 5 and 7 days, and under the inverted microscope, the morphology of the ADSCs and the proliferation on the surface of the microcarriers were assessed. The chondrocytes on the surface of the microcarrier were digested, separated and counted with a blood cell counting plate (Yancheng Glass Instrument Company, Yancheng, China), then a cell growth curve was constructed. Cell microcarrier samples were taken on day 3 to be observed by scanning electron microscopy (Hitachi S-4500; Hitachi, Tokyo, Japan), and the cell morphology and matrix secretion was observed. The static monolayer culture of ADSCs in the control group was observed to assess the ADSC morphology and proliferation at days $1,3,5$ and 7 . They were then collected and the survival rate was calculated using a trypan blue dye exclusion test. Finally , a cell growth curve was constructed based on whole cell counts.

Cytochemical and immunocytochemical analysis. The ADSCs growing on the microcarriers and those in monolayer culture were harvested, digested and smeared. They were then subjected to safranin-O and toluidine blue staining to observe the levels of glycosaminoglycan (GAG) synthesis and secretion from the extracellular matrix. The type II collagen monoclonal antibody was incubated with the cells with DAB (Beijing Hepten and Protein Biomedical Institute, Beijing, China) as the substrate, and the immunoperoxidase method was used for development. Brown staining indicated positive staining, and thus the occurrence of type II collagen secretion and synthesis.

\section{Results}

ADSC adherent growth on the surface of the Cytodex 3 microcarriers. Three hours following inoculation, a number of cells had adhered to the surface of microcarriers. After $24 \mathrm{~h}$, the majority of the cells had adhered to microcarriers, and only a small number of dead cells were present in the medium. Cells gradually changed from a spherical and hemispherical to a short fusiform shape, and occasionally there were pseudopodium with irregular shapes (Fig. 1A).

On day 3 , the number of cells on the surface of the microcarriers increased significantly, and the adherent cells were apparent on a different focal plane. Cell matrix secretion around the cells, and occasionally connecting cells between the microcarriers, were observed. Cells were spindle-shaped and flat. There were few dead cells in the medium, and cells adhered well to microcarriers (Fig. 1B). On the 5th day, the majority of the microcarrier surface was covered with cells and cells had grown in layers, with abundant extracellular matrix secretion, indistinguishable single cell morphology, and large cell connection bodies between microcarriers, whilst microcarriers displayed cluster growth (Fig. 1C). On the 7th day, microcarrier surfaces were completely covered by cells overlapping as a mass, leading to a rough, uneven surface on the microcarriers. There were widespread cell connections between the microcarriers, and there were almost no dead cells in the medium (Fig. 1D).

Growth of ADSCs in static culture. Two hours after cells were treated with the induction medium, cells began to adhere to the wall, and after $24 \mathrm{~h}$, all cells were adherent. The cells were spindle-shaped or polygonal, and were slightly larger than non-induced ADSCs. Once fully adherent, cells began to rapidly proliferate, after 3 days they reached a level of $50 \%$ fusion, and after 5 days they reached $80 \%$ fusion. Along with 

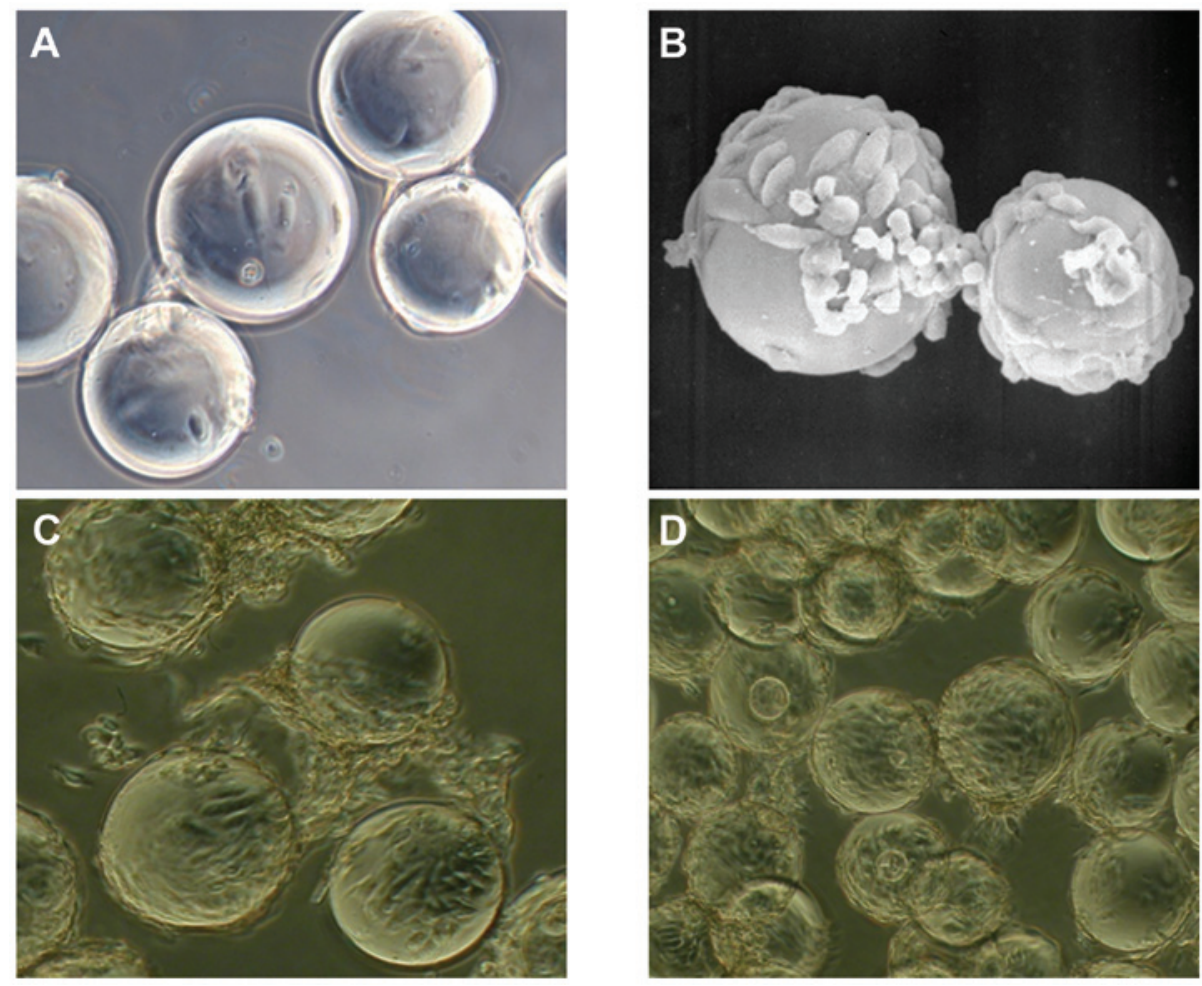

Figure 1. Morphological observation of cell microcarriers with SEM and light microscopy. (A) ADSCs cultured on microcarriers for 24 h and viewed with a light microscope (magnification, x400). Cells gradually attach to surface of the microcarrier, becoming short and fusiform. (B) ADSCs cultured on microcarriers for 3 days observed by SEM (magnification, x800). The cell number increased significantly after 3 days, and became long and fusiform in shape. (C) ADSCs cultured on microcarriers for 5 days, observed with a light microscope (magnification, x400). Cell culture on microcarriers. The majority of the microcarrier surface was covered with overlapping-growth cells and microcarriers presented cluster-style growth. (D) ADSCs cultured on microcarriers for 7 days, observed with a light microscope (magnification, $x 400$ ). Microcarriers were completely covered with overlapping cells and widespread cell connections between the microcarriers were identified. SEM, scanning electron microscopy; ADSC, adipose-derived stem cell.

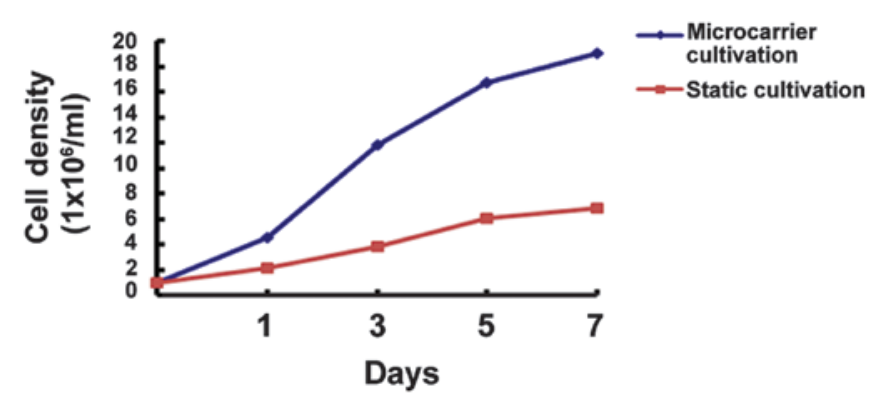

Figure 2. Cell growth curves of adipose-derived stem cells under two different culture conditions.

the increase in cell density, the proliferation slowed down, and after 7 days, almost complete fusion was reached.

Growth curves of ADSCs under two different culture modes. On days 1, 3, 5 and 7, ADSCs were harvested from the RCCS container and common culture bottle and were counted, and the growth curves were as presented in Fig. 2. The Trypan blue dye exclusion test indicated that the survival rate of ADSCs harvested from the microcarriers was $>90 \%$. According to the blood cell count, it was clear that the growth of ADSCs accelerated from day 3 after RCCS inoculation, and the cell density reached its highest value at day 7, 19 times the initial inoculation. On day 7, the total number of harvested cells cultured in a static monolayer was $\sim 6$ times the original.
Analysis of cytochemistry and immunocytochemical examination. It was demonstrated by ADSC smear staining in the two groups, that toluidine blue (Fig. 3) and safranin-O (Fig. 4) staining in the microcarrier culture was strongly positive, and was stronger than that in the static culture group. The positive staining indicated that the extracellular matrix contained a high level of GAGs; and type II collagen staining was also strongly positive (Fig. 5), indicating that the ADSCs cultured on the microcarriers through dynamic induction culture were able to express matrix components characteristic of cartilage.

\section{Discussion}

ADSCs are relatively easy to obtain and have a wider range of sources compared with bone marrow stromal stem cells. De Ugarte et al (8) identified no significant difference between bone marrow-derived stem cells and ADSCs in the time taken to obtain them, the cell growth kinetics, cell aging, gene transfection and cell adhesion properties. Thus, ADSCs have exceptional advantages and prospects as seed cells for cartilage tissue engineering.

The clinical application of cartilage tissue engineering often requires a great quantity of seed cells, and studies have demonstrated that when the cell number is $<1 \times 10^{7} / \mathrm{ml}$, it is not able to form cartilage or can only generate small amounts (9). The traditional method of amplification culture is slow and does not reliably maintain the stem cell phenotype subsequent to induction; and studies have demonstrated that appropriate 

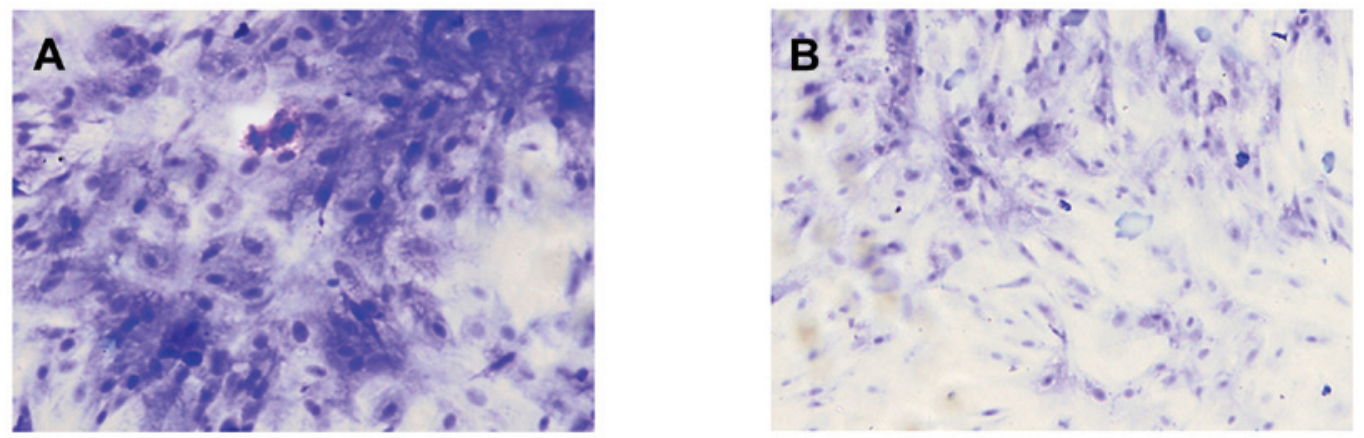

Figure 3. Toluidine blue staining to examine GAGs in the matrix. (A) Microcarrier culture-induced ADSC smear with a strong positive stain, indicating high levels of GAGs (magnification, x200). (B) Static culture-induced ADSC smear with a weak positive stain, indicating low levels of GAGs (magnification, x200). GAG, glycosaminoglycan; ADSC, adipose-derived stem cell.
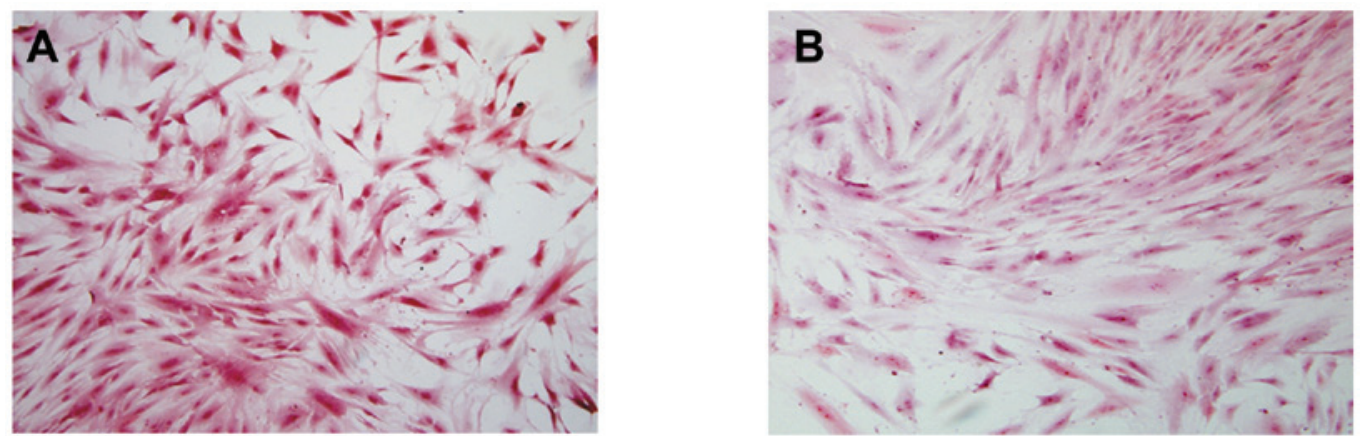

Figure 4. Safranin-O staining to examine GAGs. (A) Microcarrier culture-induced ADSC smear with a strong positive stain, suggesting high levels of GAGs (magnification, x200). (B) Static culture-induced ADSC smear with a weak positive stain, suggesting low levels of GAGs (magnification, x200). GAG, glycosaminoglycan; ADSC, adipose-derived stem cell.
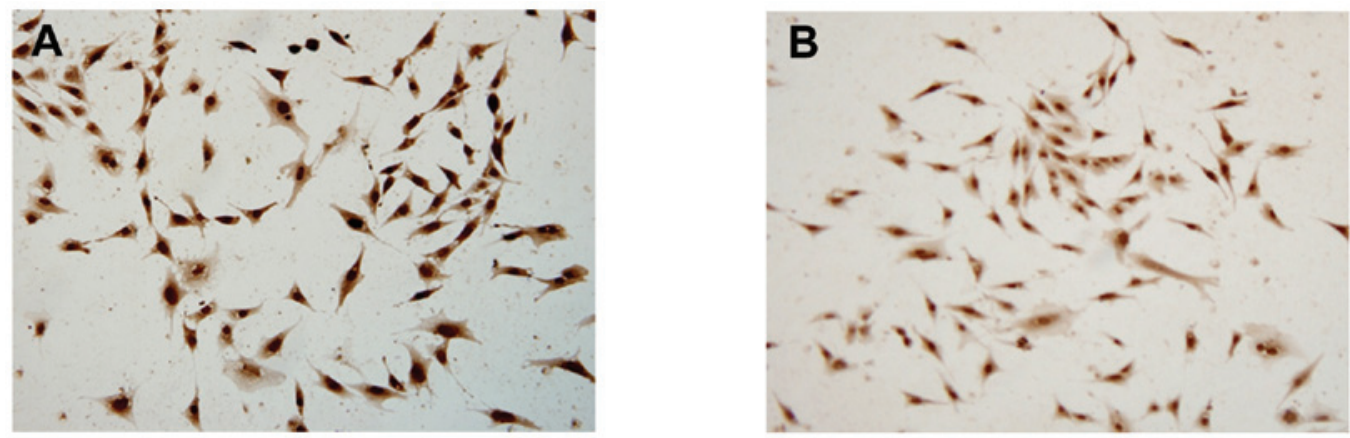

Figure 5. Immunocytochemical examination of type II collagen. (A) Microcarrier culture-induced ADSC smear. A strongly positive stain indicated high levels of type II collagen in the matrix (magnification, x200). (B) Static culture-induced ADSC smear. A weakly positive stain indicated low levels of type II collagen in the matrix (magnification, $\mathrm{x} 200$ ). ADSC, adipose-derived stem cell.

mechanical stimulation and three-dimensional culture conditions are conducive to the differentiation of ADSCs into chondrocytes, and promote the synthesis of extracellular matrix $(10,11)$; the dynamic three-dimensional culture combines the mechanical environment with the three-dimensional culture surface, to further improve the biochemical and biomechanical function of cartilage tissue engineering.

Microcarriers are widely used for the large-scale culture of attachment-dependent cells $(12,13)$, as they possess an increased surface area for cell adhesion, and thus, numerous seed cells can be harvested in a short time period (14). The microcarrier dynamic culture is the combination of using microcarriers with a bioreactor, and has been widely used in the field of tissue engineering. In the dynamic process of cultivation, external fluid mechanical stimulation is able to significantly increase cell proliferation and promote synthesis of DNA. Using the bioreactor and microcarrier technique, the cell concentration can reach $1 \times 10^{8} / \mathrm{ml}(15,16)$, which is difficult to achieve under conventional culture conditions. The microcarrier has a larger specific surface area (110 g Cytodex 3 provides $\sim 4600 \mathrm{~cm}^{2}$ surface area) (17) than a common culture flask, and it can cultivate up to hundreds of times more cells and meet the required quantities of seed cells to construct a large volume of tissue. The rotating bioreactor (RCCS) was 
also used, as the whole culture container is driven by the motor to rotate along a horizontal axis, so the culture medium and cell particles rotate together with the rotary container, the microcarrier particles can form a liquid suspension track on the horizontal axis, and the gravity, buoyancy and shear force can achieve a balance, which constitutes a microgravity environment conducive to cell aggregation. This balances the amplification of growth in each direction of the microcarriers' surface. In this dynamic environment, cells and nutrients are prone to homogeneous distribution, which can significantly improve the exchange of nutrients and metabolites throughout the microenvironment, maintaining the homogeneity and stability of the culture environment and effectively promoting cell proliferation.

Another problem facing tissue construction is how to maintain the induced phenotype, and not to allow cells to undergo dedifferentiation. The correct hydrostatic pressure and shear stress aids in maintaining the phenotype and function of cartilage cells (18-21). The use of a biological reactor and microcarrier with dynamic three-dimensional culture provides an effective environment for cell proliferation, and also the periodic mechanical stimulation required in the process of cell differentiation. This serves an important function in the mechanical environment, which can effectively promote differentiation of the stem cells into chondrocytes (22-25). In a dynamic environment, the enhanced cell activity coupled with the inductive factors, leads to increased production and secretion of proteins to form the specific extracellular matrix and promote the differentiation and maturation of cells.

In conclusion, this type of dynamic culture microenvironment provides cell aggregation, fast three-dimensional growth and effective differentiation conditions, and provides the most effective method for tissue engineering. The results of the current study confirmed this. In the present study, the ADSCs quickly attached, extended and rapidly proliferated on the microcarriers. On day 7, cells covered the surfaces of the microcarriers completely, and grew, projecting out from the microcarriers. The microcarriers connected with each other and formed masses. The number of cells in the microcarrier culture increased by almost 20 -fold in one week, while that of the static culture increased only by 6 -fold, and histochemical and immunohistochemical analysis indicated that microcarrier culture cells possessed improved phenotypes and extracellular matrix production. The current study used ADSCs as seed cells to construct cartilage tissue, providing an experimental basis, and the foundation for future investigation of cartilage tissue engineering.

\section{Acknowledgements}

The present study was supported by the 863 National Program (grant no. 2002AA205021) and the Beijing Municipal Science and Technology Commission (grant no. H060920050630).

\section{References}

1. Zuk PA, Zhu M, Mizuno H, et al: Multilineage cells from human adipose tissue: implications for cell-based therapies. Tissue Eng 7: 211-228, 2001.
2. Zuk PA, Zhu M, Ashjian P, et al: Human adipose tissue is a source of multipotent stem cells. Mol Biol Cell 13: 4279-4295, 2002.

3. Planat-Benard V, Silvestre JS, Cousin B, et al: Plasticity of human adipose lineage cells toward endothelial cells: physiological and therapeutic perspectives. Circulation 109: 656-663, 2004.

4. Ogawa R, Mizuno H, Watanabe A, et al: Osteogenic and chondrogenic differentiation by adipose-derived stem cells harvested from GFP transgenic mice. Biochem Biophys Res Commun 313: 871-877, 2004.

5. Ogawa R, Mizuno H, Watanabe A, et al: Adipogenic differentiation by adipose derived stem cells harvested from GFP transgenic mice including relationship of sex differences. Biochem Biophys Res Commun 319: 511-517, 2004.

6. Fujimura J, Ogawa R, Mizuno H, et al: Neural differentiation of adipose-derived stem cells isolated from GFP transgenic mice. Biochem Biophys Res Commun 333: 116-121, 2005.

7. Marlovits S, Tichy B, Truppe M, et al: Collagen expression in tissue engineered cartilage of aged human articular chondrocytes in a rotating bioreactor. Int J Artif Organs 26: 319-330, 2003.

8. De Ugarte DA, Morizono K, Elbarbary A, et al: Comparison of multi-lineage cells from human adipose tissue and bone marrow. Cells Tissues Organs 174: 101-109, 2003.

9. Koch RJ and Gorti GK: Tissue engineering with chondrocytes. Facial Plast Surg 18: 59-68, 2002.

10. Winter A, Breit S, Parsch D, et al: Cartilage-like gene expression in differentiated human stem cell spheroids: a comparison of bone marrow-derived and adipose tissue-derived stromal cells. Arthritis Rheum 48: 418-429, 2003.

11. Ogawa R, Mizuno S, Murphy GF and Orgill DP: The effect of hydrostatic pressure on three-dimensional chondroinduction of human adipose-derived stem cells. Tissue Eng Part A 15: 2937-2945, 2009.

12. Costa AR, Withers J, Rodrigues ME, et al: The impact of microcarrier culture optimization on the glycosylation profile of a monoclonal antibody. Springerplus 2: 25, 2013.

13. Chu L and Robinson DK: Industrial choices for protein production by large scale cell culture. Cur Opin Biotechnol 12: 180-187, 2001.

14. Rudolph G, Lindner P, Gierse A, et al: Online monitoring of microcarrier based fibroblast cultivations with in situ microscopy. Biotechnol Bioeng 99: 136-145, 2008.

15. Saris DB, Sanyal A, An KN, et al: Periosteum reponds to dynamic fluid pressure by proliferating in vitro. J Orthop Res 17: 668-677, 1999.

16. Griffiths B: Scale-up of suspension and anchorage-dependent animal cells. Mol Biotechnol 17: 225-238, 2001.

17. Dan JL, Xu JZ,Zhou Q, et al: Culturing human mesenchymal stem cells in vitro in large scale on microcarriers. Orthop J Chin 12: 1158-1160, 2004 (In Chinese).

18. Parkkinen JJ, Ikonen J, Lammi MJ, et al: Effects of cyclic hydrostatic pressure on proteoglycan synthesis in cultured chondrocytes and articular cartilage explants. Arch Biochem Biophys 300: 458-465, 1993.

19. Lammi MJ, Inkinen R, Parkkinen JJ, et al: Expression of reduced amounts of structurally altered aggrecan in articular cartilage chondrocytes exposed to high hydrostatic pressure. Biochem J 304: 723-730, 1994.

20. Simon WH, Mak A and Spirt A: The effect of shear fatigue on bovine articular cartilage. J Orthop Res 8: 86-93, 1990.

21. Tomatsu T, Imai N, Takeuchi N, Takahashi K and Kimura N: Experimentally produced fractures of articular cartilage and bone. The effects of shear forces on the pig knee. J Bone Joint Surg Br 74: 457-462, 1992.

22. van der Kraan PM, Buma $P$, van Kuppevelt $T$ and van den Berg WB: Interaction of chondrocytes, extracellular matrix and growth factors: relevance for articular cartilage tissue engineering. Osteoarthritis Cartilage 10: 631-637, 2002.

23. Dickinson SC, Sims TJ, Pittarello L, et al: Quantitative outcome measures of cartilage repair in patients treated by tissue engineering. Tissue Eng 11: 277-287, 2005.

24. Li Z, Kupcsik L, Yao SJ, Alini M and Stoddart MJ: Mechanical load modulates chondrogenesis of human mesenchymal stem cells through the TGF-beta pathway. J Cell Mol Med 14: 1338-1346, 2010.

25. Li Z, Yao SJ, Alini M and Stoddart MJ: Chondrogenesis of human bone marrow mesenchymal stem cells in fibrin-polyurethane composites is modulated by frequency and amplitude of dynamic compression and shear stress. Tissue Eng Part A 16: 575-584, 2010 . 